\title{
Memoria organizacional para el soporte de gestión documental en actividades de investigación
}

Javier De la Hoz Freyle ${ }^{1}$ Luis Carlos Gómez Flórez ${ }^{2}$

RESUMEN

La gestión de la calidad en las organizaciones supone una serie de beneficios que las impulsa a obtener ventajas competitivas a través de la mejora continua de sus procesos. Sin embargo, este enfoque de gestión administrativo conlleva una serie de barreras y dificultades que obstaculizan la rápida consecución del éxito en sus prácticas. Una de las áreas de la gestión de la calidad que ha presentado dificultades en el Centro de Estudios e Investigaciones Ambientales (CEIAM) de la Universidad Industrial de Santander (UIS) es la gestión documental, principalmente debido a la alta rotación del personal técnico, el cual está compuesto en su mayoría por estudiantes de química de últimos semestres, que abandonan el laboratorio del centro una vez culminan sus estudios universitarios, lo que obliga a la entidad a incluir nuevos estudiantes continuamente para dar soporte a sus labores de investigación y extensión, entorpeciendo así esta área de la gestión de la calidad. Como alternativa de solución a la problemática descrita se planteó y desarrolló una investigación en la cual se pretendía aplicar estrategias de gestión del conocimiento para disminuir las dificultades presentadas en la gestión documental del laboratorio del CEIAM. Esta condujo a la implementación de una memoria organizacional basada en cloud storage para el almacenamiento y recuperación de los recursos documentales propios del sistema de gestión de calidad del centro, así como la inclusión de conocimientos referentes a su utilización que sirvieran de guía al personal técnico del laboratorio. A continuación, se presenta el desarrollo de dicha investigación, así como sus principales resultados.

Palabras clave: Cloud storage. Gestión de la calidad. Gestión del conocimiento. Gestión documental. Memoria organizacional.

\section{INTRODUCCIÓN}

El Centro de Estudios e Investigaciones Ambientales (CEIAM) de la Universidad Industrial de Santander (UIS) se dedica a actividades de investigación y extensión en el área de análisis de aguas y aires con fines ambientales. Cuenta con un laboratorio exclusivo para el desarrollo de su misión de extensión, proveyéndole

1 Javier De la Hoz Freyle - Universidad Industrial de Santander - javierdlahoz@gmail.com

${ }^{2}$ Luis Carlos Gómez Flórez - Universidad Industrial de Santander - Icgomezf@uis.edu.co 
servicios de análisis de muestras a la comunidad regional, así como a la nacional, e internacional, pero también, se utiliza para la realización de diversas investigaciones en el campo de aguas residuales principalmente (CEIAM, 2010).

El personal técnico del laboratorio de aguas consta en su mayoría de estudiantes de química de últimos semestres que prestan sus servicios al CEIAM a modo de práctica empresarial o auxiliaturas. Dichos estudiantes una vez culminan sus estudios universitarios tienden a abandonar el centro, dando espacio a nuevos prospectos que quieran adquirir experiencia en trabajo en laboratorios de análisis de muestras. Esta mecánica de rotación de personal técnico ha provocado que las prácticas de gestión de la calidad en el CEIAM se vean obstaculizadas, principalmente en la gestión documental, debido al desconocimiento del nuevo personal acerca los formatos y formularios activos del centro, así como su correcto diligenciamiento.

Dicha problemática, entre otras, motivaron el desarrollo de una investigación liderada por los autores de este artículo, en la cual se incorporaron conceptos y prácticas de gestión del conocimiento en la gestión de la calidad del centro, a su vez que se utilizaron principios del paradigma cloud computing para el soporte tecnológico de una memoria organizacional, diseñada como artefacto para el almacenamiento y recuperación de recursos de conocimiento, así como para la gestión documental.

En este artículo se presenta el desarrollo de la investigación mencionada anteriormente, resaltando el producto de la memoria organizacional para el soporte de la gestión documental del CEIAM. El presente inicia con la descripción de los conceptos teóricos utilizados en la investigación, seguida de la presentación de la metodología, continuando con los resultados, y finalizando con las conclusiones.

\section{CONCEPTOS TEÓRICOS}

A continuación se describen los conceptos teóricos tenidos en cuenta en el desarrollo del presente artículo.

\subsection{PROCESOS DE LA GESTIÓN DEL CONOCIMIENTO}

En la literatura de gestión del conocimiento se encuentran diferentes definiciones de los procesos que esta debe tener para lograr sus objetivos, una de las más citadas, y la cual ha sido adoptada en el desarrollo de la investigación, es la de Alavi y Leidner (2001), que definen el ciclo de procesos como: creación; almacenamiento/recuperación; transferencia; y utilización. Ellas consideran que dichos procesos deben ser vistos de manera cíclica, partiendo de la premisa que una vez el conocimiento haya sido creado, posteriormente almacenado en fuentes donde otras personas puedan acceder a él, transferido a los individuos que lo necesiten en el momento indicado, y utilizado por parte de otros entes, este se deriva en la creación de nuevos conocimientos o en la modificación de los existentes, puesto que cada persona posee constructos y estructuras mentales que los distinguen de los demás y generarán variaciones de los conocimientos adquiridos (DE LA HOZ, CARRILLO Y GÓMEZ, 2012). En la siguiente figura se puede observar el ciclo de procesos anteriormente descrito.

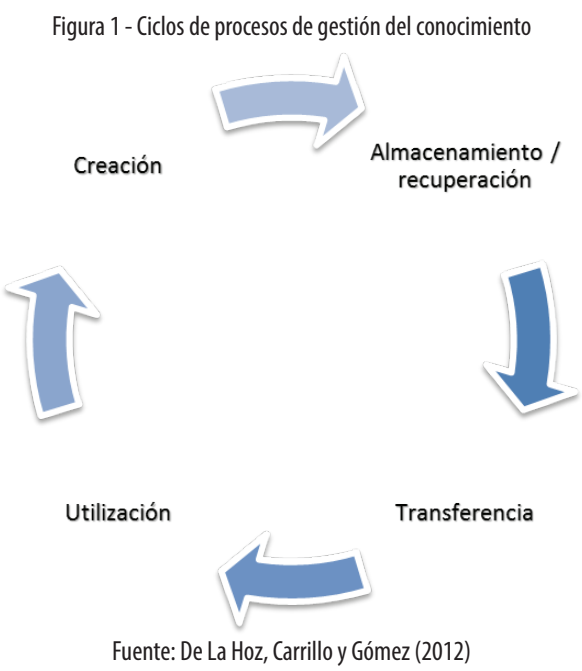


De los procesos descritos por Alavi y Liedner, la investigación se centró en el proceso de almacenamiento y recuperación, en donde según los autores, la memoria organizacional toma un rol protagónico, y es descrita como el medio tecnológico para facilitar la inserción de recursos de conocimiento y su posterior visualización en los procesos de la gestión del conocimiento.

\subsection{MEMORIA ORGANIZACIONAL}

Según Alavi y Leidner (2001) el principal soporte para el desarrollo de las actividades de almacenamiento y recuperación de los procesos de la gestión del conocimiento es la memoria organizacional, sin embargo, no fueron los primeros autores en hablar de ello, de hecho, en los trabajo de Argyris y Schon (1978) y Duncan y Weiss (1979) a finales de la década de los 70 apuntaron que "si una organización aprende, entonces, ese aprendizaje debería estar disponible en un futuro" dando un indicio que la organización debería tener algún mecanismo o artefacto para almacenar los conocimientos adquiridos para luego traerlos a disposición. Asimismo, Walsh y Ungson (1991) afirmaron que las organizaciones deberían, de cierta manera, almacenar la información y experiencias para que estas pudieran ayudar a tomar decisiones en el presente, respaldando las declaraciones de los anteriores autores.

Posteriormente, los primeros autores en utilizar el término fueron Ackerman y Malone (1990) en su trabajo denominado "Answer Garden", en él expresan que la memoria organizacional es la habilidad de la organización de beneficiarse de sus experiencias del pasado para actuar efectivamente en el presente. En ese mismo trabajo, ellos presentaron el desarrollo de una herramienta tecnológica para el almacenamiento y recuperación de recursos de conocimiento como parte de su memoria organizacional. Luego, Ackerman (1998) decide incluir como factor principal de la memoria organizacional lo que él denomina repositorios de información interna, así como manuales corporativos, bases de datos, sistemas de archivos e incluso historias del personal, definiendo así, contenedores de conocimientos en forma física y digital a lo conocido como memoria organizacional.

Adicionalmente, diversos trabajos en el área de la gestión del conocimiento exponen que una memoria organizacional basada en tecnologías de información (TI) debe contar con un directorio de personas expertas en temas específicos que pueden ser consultadas ante cualquier inquietud, además de un repositorio de documentos que pueden ser accedidos ante cualquier necesidad de conocimientos (ACKERMAN; MALONE, 1990; STEIN, 1995; BORGHOFF; PARESCHI, 1997; CONKLIN, 2001; DE LA HOZ; CARRILLO; GÓMEZ, 2013b).

Abecker et al (1997) consideran que las memorias organizacionales, además de las tareas de almacenamiento y recuperación propuestas por Alavi y Leidner (2001), renombradas por ellos cómo identificación, adquisición y presentación de conocimiento, deben facilitar la diseminación, utilización y desarrollo de conocimiento, procesos los cuales son encapsulados por Alavi y Liedner, en transferencia y aplicación (ver figura 2). De igual manera, Abecker et al (1997) exponen que, es necesario soportar el diseño, desarrollo, y utilización de las mismas en tecnologías de información. Trabajos como los propuestos por Alavi y Leidner (2001), Borghoff y Pareschi (1997), Gandon (2004), y Vasconcelos, Gouveia y Kimble (2002), entre otros, afirman tal planteamiento. Por lo tanto, las memorias organizacionales están basadas en un enfoque social, administrativo, y tecnológico.

Figura 2 - Modelo de Memoria Organizacional

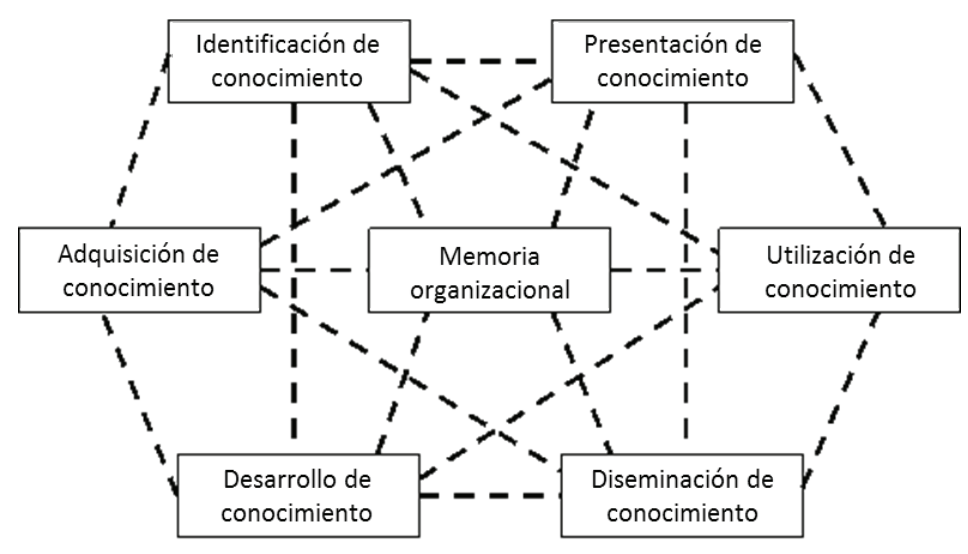

Fuente: Abecker et al (1997) 


\section{METODOLOGÍA}

La metodología utilizada en el desarrollo del proyecto que condujo a la implementación de una memoria organizacional para el soporte de la gestión documental del CEIAM fue la Metodología de Sistemas Blandos (SSM) propuesta por Checkland y Scholes (1999). Esta metodología se basa en el enfoque de investigación-acción, y cuenta con siete (7) etapas cíclicas para mejorar lo que ellos denominan "situación considerada problemática". En la figura 3 se puede observar el esquema de la SSM.

En ella se encuentran los roles tomados por los autores del presente, los cuales se consideran como los mejoradores de la situación. De igual manera, la historia a la que hace referencia la SSM es el estudio previo de la literatura sobre los ejes temáticos de la investigación desarrollada, para el caso presentado: gestión del conocimiento; gestión de la calidad; gestión documental; cloud computing; memorias organizacionales; y sistemas de información.

En el análisis de la situación como una cultura, se hicieron acercamientos a la situación considerada problemática teniendo en cuenta los sistemas sociales y políticos del centro, así como el problema en sí. Esto ayudó a determinar las dificultades de la organización respecto a la gestión de la calidad, identificando unas áreas estratégicas a ser tratadas por la gestión del conocimiento, entre las cuales se encontró la gestión documental, los resultados de este análisis se encuentran ampliados en el trabajo de De La Hoz, Carrillo y Gómez (2013a).

Figura 3 - Metodología de Sistemas Blandos

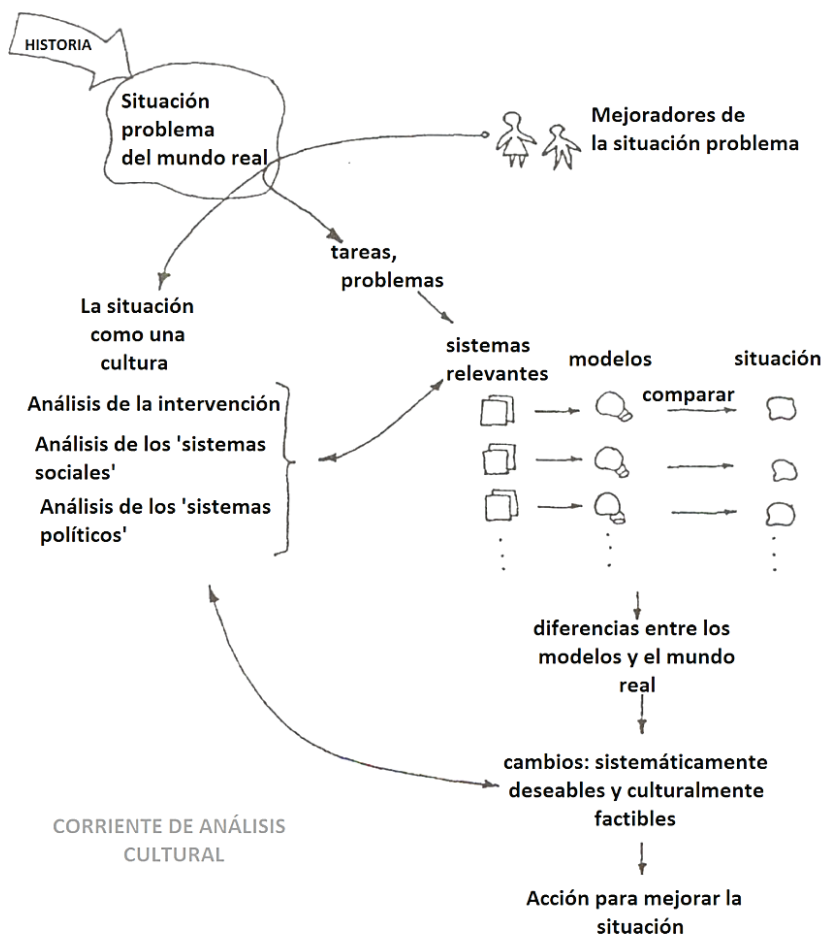

CORRIENTE DE ANÁLISIS

LÓGICO

Fuente: Checkland y Scholes (1999)

Una vez realizado el análisis de la situación como una cultura, se elaboraron los nombramientos de lo que el autor de la SSM denomina "sistemas relevantes de actividad con propósito", que es la descripción general del sistema que contribuirá a la mejora de la situación considerada problemática. En ellos se plasmaron las ideas de tratar la gestión de la calidad por medio de la gestión del conocimiento para disminuir las dificultades presentadas en el CEIAM.

Posteriormente se modelaron los sistemas relevantes de actividad con propósito y se hicieron una serie de debates, en los cuales, se determinaron cambios que permitieron al sistema encajar con la cultura de la organización y ser, como el autor lo denomina "sistemáticamente deseables y culturalmente factibles". 
Habiendo definido los cambios del sistema, se desarrollaron las acciones para mejorar la situación, las cuales consistieron en la implementación de varios sistemas administrativos y tecnológicos, entre ellos la memoria organizacional para el soporte de la gestión documental, la cual se presenta en el siguiente aparte.

\section{RESULTADOS: MEMORIA ORGANIZACIONAL PARA EL SOPORTE DE LA GESTIÓN DOCUMENTAL}

Cloud storage según Bowers, Juels y Oprea (2009), es una familia de servicios de manejo de archivos en línea cuya popularidad va en aumento utilizando simples interfaces que permiten abstraer las complejidades del manejo del hardware. Entre los servicios más comunes de cloud storage se encuentran: Dropbox; Google Drive; Box; JoliCloud; SkyDrive; entre otros. Como se menciona anteriormente, la memoria organizacional debe estar basada en un enfoque administrativo, social y tecnológico. En cuanto a la parte tecnológica se optó por el uso de cloud computing, más precisamente cloud storage para así conseguir elasticidad en el consumo de recursos, así como la posibilidad que estos fueran accedidos desde cualquier lugar y momento, y desde cualquier dispositivo con conexión a internet, a la vez que se facilita el trabajo al desarrollador en cuanto a la realización de modificaciones y actualizaciones, según los trabajos Ahmad-Bhat et al (2010); Armbrust et al (2010); Marks y Lozano (2010); Nagaprasad et al (2010); y Nist (2011).

Como se expresa anteriormente, la labor principal de una memoria organizacional es el almacenamiento y recuperación de recursos de conocimiento (ABECKER ET AL, 1997; ALAVI Y LEIDNER, 2001; CONKLIN, 2001). Teniéndolo presente, para el diseño del modelo de la memoria organizacional, se plantearon dos (2) subsistemas: uno de almacenamiento de recursos de conocimiento; y otro de recuperación de recursos de conocimiento.

El subsistema de almacenamiento de recursos de conocimiento es el encargado de capturar y guardar todos los recursos de conocimientos de la gestión documental, así como los formularios y formatos utilizados en los procedimientos de análisis de muestras. La labor de la herramienta tecnológica basada en cloud storage es el almacenamiento de recursos de conocimiento de manera segura, permitiendo el acceso a las personas que deben hacer uso de los mismos, y proveyendo mecanismos de indexación y de inserción de metadatos, a través de una ontología.

En los trabajos de Fensel et al (2000), Gandon (2004), Harris et al (2004), Guarino (1998) y Noy y Mcguinness (2000) se expresa que, las ontologías facilitan las comunicación entre los seres humanos y sistemas computacionales, proveyendo conceptualización en los dominios de conocimiento. Asimismo, en los de Almeida y Barbosa (2009), Staab et al (2001), Stevens, Goble y Bechhofer (2000) y Weinstein (1997), se concluye que el uso de ontologías para la inserción de metadatos en recursos de conocimiento puede mejorar en gran manera los sistemas de búsqueda y transferencia de conocimiento. De hecho, en el trabajo de Vasconcelos, Gouveia y Kimble (2002) se puede observar como a través del uso de ontologías se soporta un sistema de memoria organizacional para un proyecto de gestión del conocimiento. Razones por las cuales se optó por incluir una ontología como base para inserción de metadatos en la memoria organizacional. El diseño y elaboración de la ontología se puede encontrar en el trabajo de De La Hoz y Gómez (2013).

La principal funcionalidad del subsistema de recuperación de conocimiento, es como su nombre lo indica poder recuperar los recursos de conocimiento, formularios, y formatos, almacenados en la memoria organizacional, para que puedan ser utilizados en la transferencia, aplicación y generación de conocimientos, usando como base para la búsqueda lo metadatos encapsulados en la ontología.

De esa manera la memoria organizacional permite almacenar los archivos requeridos por el sistema de gestión documental del CEIAM, de tal manera que exista una base de formularios, formatos, manuales, buenas prácticas, instructivos y demás que puedan ser consultados y accedidos por el personal administrativo y técnico del mismo. De igual forma, cada formulario o formato que hace parte de los procedimientos de análisis de muestras, posee un campo de metadatos específico para la inserción de instrucciones referente a su diligenciamiento y uso, así, cada vez que un estudiante encargado de una función técnica tenga que hacer uso de un formulario, puede encontrar las instrucciones de diligenciamiento, disminuyendo la posibilidad de error por desconocimiento.

Además, la memoria organizacional maneja un sistema de versiones que impide que las versiones obsoletas y desactualizadas hagan parte de los listados de documentos habilitados para el uso del personal técnico. De esa manera, se evita que los estudiantes utilicen documentos obsoletos o versiones antiguas, y se obliga a que hagan uso de la versión más reciente, manteniendo actualizado el sistema de gestión de la calidad, e impidiendo dificultades referentes a la gestión documental en las auditorías internas y externas a las que se somete el laboratorio de aguas del CEIAM.

A continuación se muestran capturas de pantalla de los principales formularios de la herramienta 
tecnológica desarrollada para la memoria organizacional para el soporte a la gestión documental en el laboratorio de aguas del CEIAM. En la figura 4 se puede observar la presentación inicial, la cual consta de un sistema de búsqueda a través de los metadatos de los recursos, la lista de los documentos disponibles de acuerdo a la categoría definida por la ontología, y las opciones de administración de usuario.

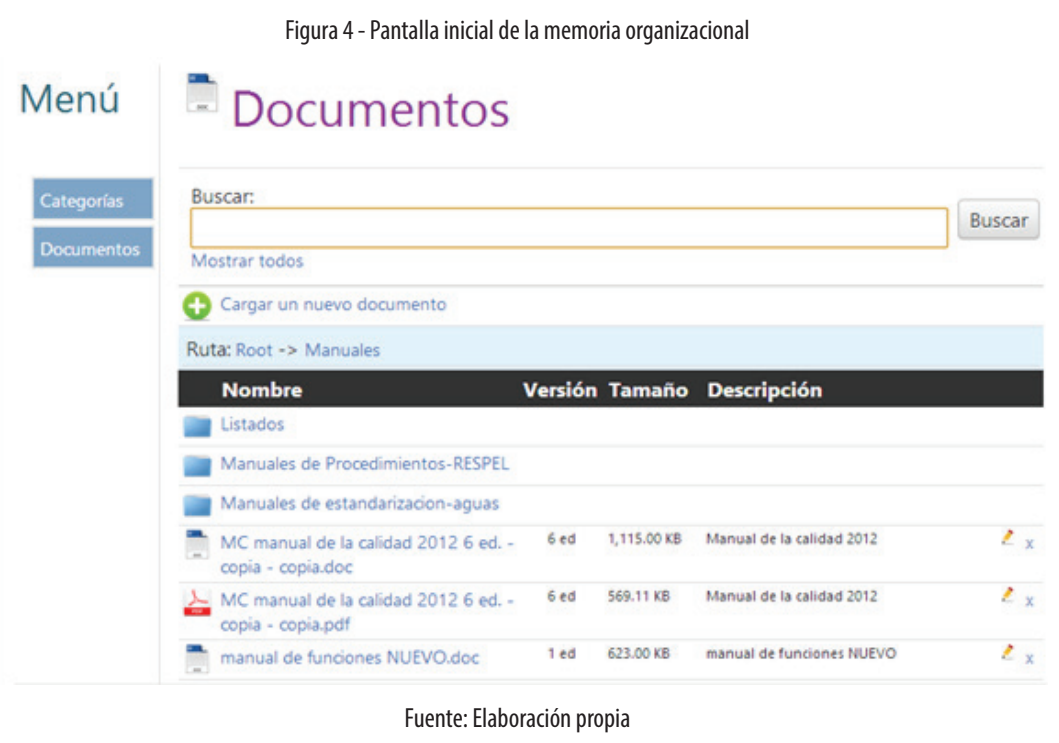

En la figura 5 se observa el formulario de vista de recursos, el cual permite consultar los metadatos e instrucciones, y descargar el archivo adjunto según los permisos que se le hayan asignado al usuario.

Figura 5 - Formulario de vista de recursos

PC manual de la calidad 20126 ed. - copia - copia.pdf
Pitulo: $\quad$ Manual de la calidad 2012
$\begin{aligned} & \text { Version: } \\ & \text { Codigo: }\end{aligned}$
$\begin{aligned} & \text { Fechation dedf } \\ & \text { vigencia: }\end{aligned}$
$\begin{aligned} & \text { Fecha de } \\ & \text { revision: }\end{aligned}$
$\begin{aligned} & \text { Es copia } \\ & \text { controlada?: }\end{aligned}$

\section{Comentarios/Instrucciones}

Fuente: Elaboración propia

Una vez desarrollada, la herramienta tecnológica fue implantada en el laboratorio de aguas del CEIAM, teniendo en cuenta las modificaciones a los procesos existentes pertinentes para el funcionamiento adecuado de la misma. Con esto se espera en un futuro, los nuevos estudiantes que ingresen al rol de personal técnico en el centro tengan una base de recursos de conocimientos que los ayude con el diligenciamiento de formularios y formatos referentes al análisis de muestras. 


\title{
5 CONCLUSIONES
}

Se espera que el continuo uso de la herramienta tecnológica desarrollada para la memoria organizacional conlleve a la disminución de errores por desconocimiento sobre el diligenciamiento de formularios y formatos referentes a los procesos de análisis de muestras del laboratorio de aguas del CIEAM, debidos a la alta rotación de personal técnico de la institución.

Para próximos trabajos se adicionará la opción de comentarios, sugerencias y observaciones de parte del personal técnico a los documentos, permitiendo tener retroalimentación que puede servir para la mejora de los formularios y formatos, así como a la mejora continua de los procesos de análisis de muestras del laboratorio, contribuyendo así al sistema de gestión de calidad.

Gracias a la investigación realizada, se concluyó que la gestión de la calidad posee ciertas barreras y dificultades que pueden ser disminuidas a través de la gestión del conocimiento. Sin embargo, esta no representa la única alternativa para tratar de mejorar la gestión de la calidad en las organizaciones, puesto que, así como lo mencionan en sus trabajos Schaffer y Thomson (1992), Young (1992), Galandere-Zile, Vanags y Kirikova (2002) y Brislan (2008) existen diversos factores que pueden obstaculizar la gestión de la calidad, en los que la falta de prácticas y estrategias de gestión del conocimiento solo forman parte de ellos.

\section{ORGANIZATIONAL MEMORY FOR SUPPORT OF DOCUMENT MANAGEMENT SYSTEM ON RESEARCH ACTIVITIES}

\begin{abstract}
Quality management on organizations implies a number of benefits which encourage them to get competitive advantages through continuous improvement of their processes. Nevertheless, this administrative approach comes with a set of barriers and difficulties which hold back the fast successful outcomes regarding its practices. One of the quality management areas which has presented some difficulties on the Centro de Estudios e Investigaciones Ambientales (CEIAM) of the Universidad Industrial de Santander (UIS) is the document management system, mainly due to the high technical staff turnover, that is largely made up of chemistry students of the last semesters, who drop out of the center laboratories as they finish their undergraduate studies, making the entity to incorporate new students continuously to support the research and development work, obstructing this quality management area. As an alternative solution to the problems outlined, we proposed and developed a research, in which we intended to apply knowledge management strategies to decrease the document management difficulties of the CEIAM's laboratory. This research led to the organizational memory implementation based on cloud storage for storage and recovery of documentary resources of the center's quality management, as well as the inclusion of knowledge concerning to its use which it could serve as guide to the technical staff of the laboratory. This paper presents the research development, as well as its main results.
\end{abstract}

Keywords: Cloud storage. Quality management. Knowledge management. Document management. Organizational memory. 


\section{REFERENCIAS}

ABECKER, A. et al. Towards a Well-Founded Technology For Organizational Memories. AAAI, Kaiserslautern, German, p.1-7, 1997.

ACKERMAN, M. S. Augmenting Organizational Memory: A Field Study of Answer Garden. ACM Transactions on Information Systems, Pittsburgh, PA, v. 16, n. 3, p. 203-224, 1998.

ACKERMAN, M. S.; MALONE, T. Answer Garden: A Tool for Growing Organizational Memory. ACM Conference on Office Information Systems, 1990, Cambridge, MA. Proceedings... Cambridge: ACM, 1990. p.31-39.

AHMAD-BHAT, M. et al. Cloud Computing: A Solution to Information Support Systems (ISS). International Journal of Computer Applications, New York, v. 11, n. 5, p. 5-9, Dec. 2010.

ALAVI, M.; LEIDNER, D. Review: Knowledge Management and Knowledge Management Systems: Conceptual Foundations and Research Issues. MIS Quarterly, Minneapolis, MN, v. 25, n. 1, p. 107-136, 2001.

ALMEIDA, M. B.; BARBOSA, R. R. Ontologies in knowledge management support: A case study. Journal of the American Society for Information Science and Technology, Silver Spring, MD, v. 60, n. 10, p. 20322047,2009

ARGYRIS, C.; SCHON, D. A. Organizational Learning: A theory of action perspective. Reading, MA: Addison-Wesley, 1978.

ARMBRUST, M. et al. A View of Cloud Computing. Communications of the ACM, Pittsburgh, PA, v. 53, n. 4, p. $50-58,2010$.

BORGHOFF, U. M.; PARESCHI, R. Information Technology for Knowledge Management. Journal of Universal Computer Science, Graz, Austria, v. 3, n. 8, p. 1-8, 1997.

BOWERS, K. D.; JUELS, A.; OPREA, A. HAIL: a high-availability and integrity layer for cloud storage. ACM conference on Computer and communications security, 16., 2009, New York. Proceedings of... New York: ACM, 2009. p.1-12.

BRISLAN, T. Difficulties with the ISO quality certification. [2008]. Availabel in: <http://www. centrorisorse.org/>. Access: 15 out. 2013.

CEIAM. Centro de Estudios e Investigaciones Ambientales. [2010]. Disponible: <http://ceiam.uis.edu.co/ >. Acceso: 15 out. 2013.

CONKLIN, J. Designing Organizational Memory: Preserving Intellectual Assets in a Knowledge Economy. In: INSTITUTE, C. (Ed.). Designing Organizational Memory: CogNexus Institute, 2001. p.2-41.

CHECKLAND, P.; SCHOLES, J. Soft Systems Methodology in Action. Lancaster, UK: John Wiley \& Sons, 1999.

DE LA HOZ, J.; CARRILLO, E.; GÓMEZ, L. C. Gestión de la calidad y del conocimiento: dos enfoques complementarios. Administer, Medellín, Colombia, n. 21, p. 71-85, jul./dez. 2012.

DE LA HOZ, J.; CARRILLO, E.; GÓMEZ, L. C. Investigación-acción aplicada a la gestión del conocimiento a través de la metodología de sistemas blandos. Lisboa, Portugal: CISTI, 2013a.

DE LA HOZ, J.; CARRILLO, E.; GÓMEZ, L. C. Memorias organizacionales en los tiempos de Cloud Storage. Tecnura, Bogotá, Colombia, v. 37, 2013b. 
DE LA HOZ, J.; GÓMEZ, L. C. Ontologías aplicadas en la gestión del conocimiento y calidad: un caso organizacional. Congreso Internacional de Evaluación en la Calidad de la Educación y de la Investigación, 2013, Bucaramanga, Colombia. Anais... Bucaramanga: UDI, 2013.

DUNCAN, R.; WEISS, A. Organizational learning: implications for organizational design. Research in Organizational Behavior, Berkeley, CA, v. 1, p. 75-124, 1979.

FENSEL, D. et al. On-To-Knowledge: Ontology-based Tools for Knowledge Management. eBusiness and eWork Conference, 2000. Cheshire Henbury: EMMSEC, 2000.

GALANDERE-ZILE, Imandra; VANAGS, Janis; KIRIKOVA, Marite. Towards knowledge management system for quality management: Improving effectivity of organisations. In: Proceedings of the Baltic Conference, BalticDB\&IS 2002-Volume 2. Institute of Cybernetics at Tallin Technical University, 2002. p. 27-38.

GANDON, F. Engineering an Ontology for a Multi-Agents Corporate Memory System. Sophia Antipolis, France: INRIA, 2004.

GUARINO, N. Formal Ontology and Information Systems. Trento, Italia: FOIS, 1998.

HARRIS, M. A. et al. The Gene Ontology (GO) database and informatics resource. Nucleic acids research, v. 32, n. Database issue, p. D258-61, 2004.

MARKS, E.; LOZANO, B. Executives Guide to Cloud Computing. New York, NY: Jhon Wisley\& Sons, 2010.

NAGAPRASAD, S. et al. Reviewing Some Platforms in Cloud Computing. International Journal of Engineering and Technology, Jordan, v. 2, n. 5, p. 348-353, 2010.

NIST. The Nist Definition of Cloud Computing. National Institute of Standards and Technology. Gaithersburg, MD: NIST, 2011.

NOY, N. F; MCGUINNESS, D. L. Ontology Development 101: A Guide to Creating Your First Ontology. Stanford: Stanford University, 2000.

SCHAFFER, R. H.; THOMSON, H. A. Successful Change Programs Begin with Resuits. Harvard Business Rev., Cambridge, MA, v. January-February, p. 80-89, 1992.

STAAB, S. et al. Knowledge processes and ontologies. Intelligent Systems, IEEE, Los Alamitos, CA, v. 16, n. 1, p. 26-34, 2001.

STEIN, E. W. Organizational Memory: Review of Concepts and Recommendations for Management. International Journal of Information Management, Hong Kong, v. 15, n. 1, p. 17-32, 1995.

STEVENS, R.; GOBLE, C. A.; BECHHOFER, S. Ontology-based Knowledge Representation for Bioinformatics. Briefing in Bioinformatics, Oxford, Inglaterra, v. 1, n. 4, p. 398-414, 2000.

VASCONCELOS, J. B.; GOUVEIA, F. R.; KIMBLE, C. An Organisational Memory Information System using Ontologies. Conferência da Associação Portuguesa de Sistemas de Informação, 3., 2002, Coimbra. Anais... University of Coimbra, 2002. p.1-17.

WALSH, J. P.; UNGSON, G. R. Organizational Memory. The Academy of Management Review, Briarcliff Manor, NY, v. 16, n. 1, p. 57-91, 1991.

WEINSTEIN, P. C. Ontology-Based Metadata. Michigan: University of Michigan Digital Library. 1997.

YOUNG, M. A framework for the successful adoption of Japanese manufacturing techniques in the United States. Academy of Management Review, Briarcliff Manor, NY, v. 17, n. 4, p. 677-700, 1992. 